\title{
A self-linking invariant of virtual knots
}

\author{
by
}

\author{
Louis H. Kauffman (Chicago, IL)
}

\begin{abstract}
This paper introduces a self-linking invariant for virtual knots and links, and relates this invariant to a state model called the binary bracket, and to a class of coloring problems for knots and links that include classical coloring problems for cubic graphs.
\end{abstract}

1. Introduction. In this paper we introduce a new invariant of virtual knots and links that is non-trivial for many virtuals, but is trivial on classical knots and links. The invariant will initially be expressed in terms of a relative of the bracket polynomial [5], and then extracted from this polynomial in terms of its exponents, particularly for the case of knots. This analog of the bracket polynomial will be denoted $\{K\}$ (with curly brackets) and called the binary bracket polynomial. See Section 3 for the definition and properties of the binary bracket. The key to the combinatorics of this invariant is an interpretation of the state sum in terms of 2-colorings of the associated diagrams.

We define the invariant

$$
\Lambda(L)=\{L\} / \sum_{O \in O(L)} A^{w\left(L^{O}\right)}
$$

for an unoriented virtual link $L$. Here $\{L\}$ denotes the binary bracket, and $\sum_{O \in O(L)} A^{w\left(L^{O}\right)}$ denotes the sum over all orientations of $L$ of the terms $A^{w\left(L^{O}\right)}$, where $w\left(L^{O}\right)$ denotes the writhe of $L$ with the specific orientation $O$. When $\Lambda(L)$ is not equal to 1 , the virtual link $L$ is non-trivial and nonclassical. See Theorem 2 of Section 3.

In the case of knots, the invariant we extract will be denoted by $J(K)$ for a virtual knot $K$. It is defined as follows. Let $w(K)$ denote the writhe of $K$. (This is the sum of the crossing signs for any orientation of the knot $K$.) A crossing $i$ in a knot $K$ is said to be odd if one encounters an odd number of classical crossings in walking along the diagram on one full path that

2000 Mathematics Subject Classification: Primary 57M25. 
starts at $i$ and returns to $i$. Let $\operatorname{Odd}(K)$ denote the set of odd crossings in the diagram $K$. In a classical diagram $K$, the set $\operatorname{Odd}(K)$ is empty. The invariant $J(K)$ is equal to the sum of the signs of the crossings in $\operatorname{Odd}(K)$, and we write

$$
J(K)=\left.w(K)\right|_{\operatorname{Odd}(K)} .
$$

$J(K)$ is invariant under equivalence of virtual knots, and hence is a selflinking number for virtuals. If $K^{*}$ denotes the mirror image of $K$, obtained by switching all the classical crossings of $K$, then $J\left(K^{*}\right)=-J(K)$. Thus $J(K)$ can detect the difference between virtual knots and their mirror images when it is non-zero. Since $J(K)$ is zero on classical knots, it detects nonclassicality when it is non-zero.

The invariants discussed in this paper are elementary. It is particularly striking that the invariant $J(K)=\left.w(K)\right|_{\operatorname{Odd}(K)}$ is only infinitesimally more complicated than the classical writhe, and yet can be used to detect nontriviality, non-classicality and chirality for infinitely many virtual knots.

The paper is organized as follows. In Section 2 we review facts and definitions about virtual knot theory. The binary bracket is introduced in Section 3; also proofs of invariance, examples and the definitions and theorems about invariants extracted from the binary bracket are given here. Section 4 delineates a collection of examples of applications of the invariants $J(K)$ and $\Lambda(L)$. These include a persistent virtual tangle, a virtual Whitehead link and virtual Borrommean rings. Section 5 introduces a combinatorial generalization of the binary bracket to an $n$-ary bracket (that is well defined on diagrams, but not an invariant of virtual links) with associated subtle coloring problems for flat virtual shadow diagrams. These coloring problems are direct generalizations of the 2-colorings associated with the binary bracket. We then explore the existence of uncolorables (there are uncolorable links even in the case $n=2$ ) for $n$ greater than or equal to 3 . We show that for $n=3$ the coloring problem defined here is directly related to the four color theorem in the form of three colorings of the edges of a cubic graph. The section ends with an explanation of the translation between these subjects.

Acknowledgements. Much of this effort was sponsored by the Defense Advanced Research Projects Agency (DARPA) and Air Force Research Laboratory, Air Force Materiel Command, USAF, under agreement F30602-01-2-05022. The U.S. Government is authorized to reproduce and distribute reprints for Government purposes notwithstanding any copyright annotations thereon. The views and conclusions contained herein are those of the author and should not be interpreted as necessarily representing the official policies or endorsements, either expressed or implied, of the Defense Advanced Research Projects Agency, the Air Force Research Laboratory, or the U.S. Government. It gives the author great pleasure to acknowledge 
support from NSF Grant DMS-0245588, and to give thanks to the University of Waterloo and the Perimeter Institute in Waterloo, Canada for their hospitality during the preparation of this research. The author is pleased to thank Bruce Richter for a helpful conversation about perfect matching.

2. Virtual knot theory. Virtual knot theory is an extension of classical diagrammatic knot theory. In this extension one adds a virtual crossing (see Figure 2) that is neither an over-crossing nor an under-crossing. A virtual crossing is represented by two crossing arcs with a small circle placed around the crossing point.

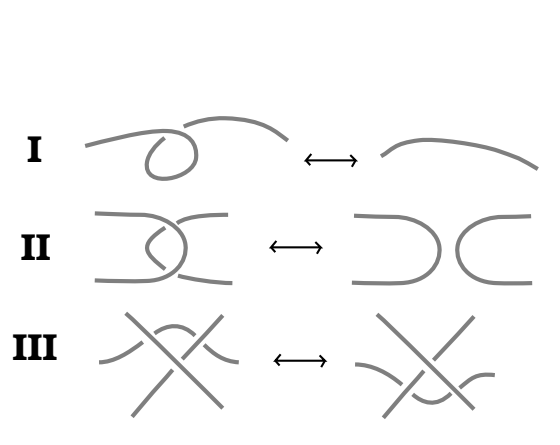

Fig. 1. Reidemeister moves

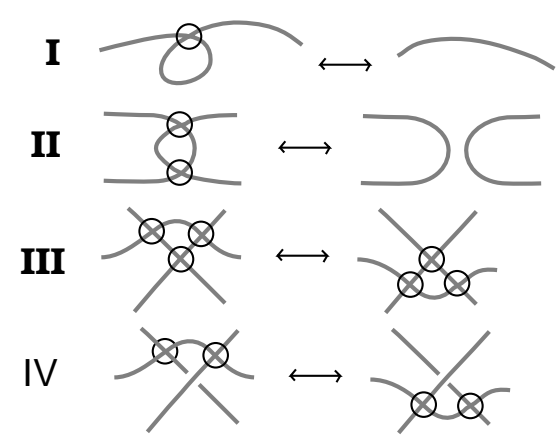

Fig. 2. Virtual moves

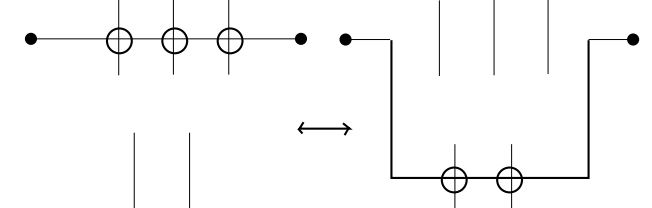

Fig. 3. The detour move

Moves on virtual diagrams generalize the Reidemeister moves for classical knot and link diagrams. See Figures 1, 2 and 3. One can summarize the moves on virtual diagrams by saying that the classical crossings interact with one another according to the usual Reidemeister moves. One adds the detour moves for consecutive sequences of virtual crossings and this completes the description of the moves on virtual diagrams. It is a consequence of the moves in Figure 2 that an arc going through any consecutive sequence of virtual crossings can be moved anywhere in the diagram keeping the endpoints fixed; the places where the moved arc crosses the diagram become new virtual crossings. This replacement is the detour move, and is illustrated schematically in Figure 3. Note that the fourth move in Figure 2 is a local detour move. (The corresponding moves with two classical crossings and one virtual crossing are not allowed.) 
One way to understand the meaning of virtual diagrams is to regard them as representatives for oriented Gauss codes (Gauss diagrams) [7, 3]. Virtual equivalence is the same as the equivalence relation generated on the collection of oriented Gauss codes modulo an abstract set of Reidemeister moves on the codes. The abstract Reidemeister moves on oriented Gauss codes correspond exactly to Reidemeister moves on diagrammatic representations of these codes in the plane (with virtual crossings), plus the use of the moves on virtual crossings (all consequences of the detour move). These extra moves make the particular choice of virtual crossings in a planar representation irrelevant. We know [7, 3] that classical knot theory embeds faithfully in virtual knot theory. That is, if two classical knots are equivalent through moves using virtual crossings, then they are equivalent solely via standard Reidemeister moves.

Virtual knots have a special diagrammatic theory that makes handling them very similar to the handling of classical knot diagrams. With this approach, one can generalize many structures in classical knot theory to the virtual domain, and use the virtual knots to test the limits of classical problems such as the question whether the Jones polynomial detects knots. Counterexamples to this conjecture exist in the virtual domain. The simplest example is the code $C=\mathrm{o} 1+\mathrm{u} 2+\mathrm{o} 3-\mathrm{u} 1+\mathrm{o} 2+\mathrm{u} 3-$ (here "o" stands for "over", "u" for "under", plus and minus signs refer to the orientations of the crossings $1,2,3$ ) a virtualized trefoil, non-trivial, but with unit Jones polynomial. It is an open problem whether one of these counterexamples is equivalent to a classical knot.

There is a useful topological interpretation for this virtual theory in terms of embeddings of links in thickened surfaces. Regard each virtual crossing as a shorthand for a detour of one of the arcs in the crossing through a 1-handle that has been attached to the 2-sphere of the original diagram. By interpreting each virtual crossing in this way, we obtain an embedding of a collection of circles into a thickened surface $S_{g} \times \mathbb{R}$, where $g$ is the number of virtual crossings in the original diagram $L, S_{g}$ is a compact oriented surface of genus $g$ and $\mathbb{R}$ denotes the real line. We say that two such surface embeddings are stably equivalent if one can be obtained from the other by isotopy in the thickened surfaces, homeomorphisms of the surfaces and the addition or subtraction of empty handles. Then we have the

Theorem $([7,8,1]$ see also [11]). Two virtual link diagrams are equivalent if and only if their corresponding surface embeddings are stably equivalent.

3. The binary bracket polynomial. In this section we define a variant of the bracket polynomial [5], called the binary bracket polynomial and denoted by $\{K\}=\{K\}(A)$ for any (unoriented) virtual knot or link $K$. 
We first describe the binary bracket as a state summation. In this respect, it has almost exactly the same formalism as the standard bracket polynomial, except that the value of an unlabeled loop is equal to 2 , and the loops in each state are colored with the colors from the set $\{0,1\}$ in such a way that the colors appearing at a smoothing are always different. This restricts the possible states to a very small number and causes the invariant to behave differently on virtual links than it does on classical links.

Let $K$ be any unoriented (virtual) link diagram. Define an unlabeled state, $S$, of $K$ to be a choice of smoothing for each classical crossing of $K$. There are two choices for smoothing a given crossing, and thus there are $2^{N}$ unlabeled states of a diagram with $N$ classical crossings. A labeled state is a state $S$ such that the labels 0 (zero) or 1 (one) have been assigned to each component loop in the state.

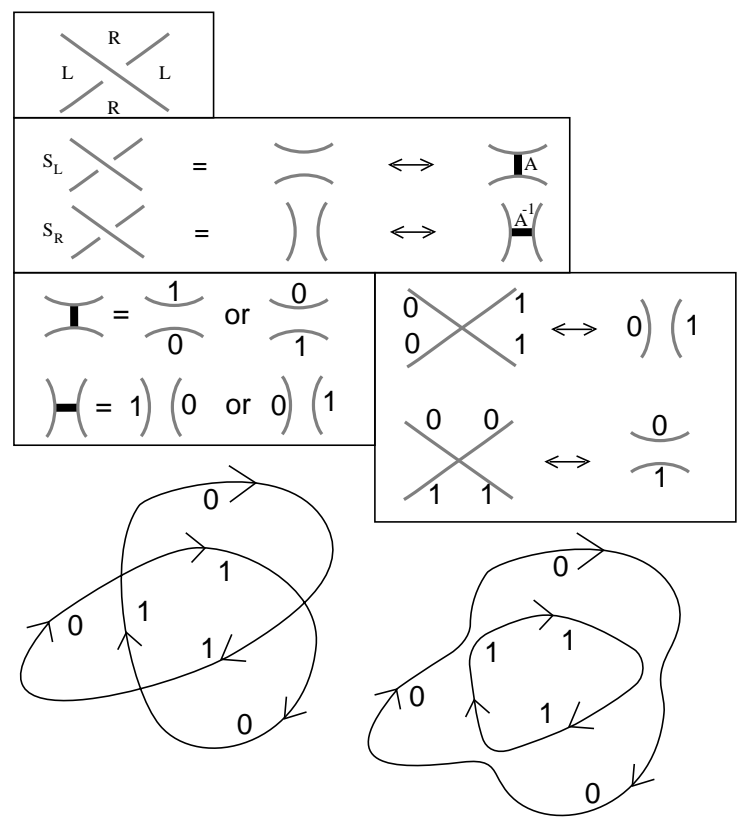

Fig. 4. Bracket smoothings

In a state we designate each smoothing with $A$ or $A^{-1}$ according to the left-right convention shown in Figure 4. This designation is called a vertex weight of the state. We require of a labeled state that the two labels that occur at a smoothing of a crossing are distinct. This is indicated by a bold line between the arcs of the smoothing as illustrated in Figure 4. Labeled states satisfying this condition at the site of every smoothing will be called properly labeled states. If $S$ is a properly labeled state, we let $\{K \mid S\}$ denote the product of its vertex weights, and we define the two-color bracket 
polynomial by

$$
\{K\}=\sum_{S}\{K \mid S\}
$$

where $S$ runs through the set of properly labeled states of $K$.

It follows from this definition that $\{K\}$ satisfies the equations

$$
\begin{gathered}
\{\backslash\}=A\{Y\}+A^{-1}\{\supset=C\}, \\
\{K \amalg O\}=2\{K\}, \quad\{O\}=2 .
\end{gathered}
$$

The first equation expresses the fact that the entire set of states of a given diagram is the union, with respect to a given crossing, of those states with an $A$-type smoothing and those with an $A^{-1}$-type smoothing at that crossing. In the first equation, we indicate that the colors at the smoothing are different by the dark band placed between the arcs of the smoothing. The second and the third equations are clear from the formula defining the state summation.

The binary bracket polynomial, $\{K\}=\{K\}(A)$, assigns to each unoriented (virtual) link diagram $K$ a Laurent polynomial in the variable $A$.

In computing the binary bracket, one finds the following behaviour under Reidemeister move I:

$$
\{\gamma\}=A\{\smile\} \quad \text { and } \quad\{\bar{\gamma}\}=A^{-1}\{\smile\}
$$

where $\gamma$ denotes a curl of positive type as indicated in Figure 6 , and $\bar{\gamma}$ indicates a curl of negative type, as also seen in this figure. The type of a curl is the sign of the crossing when we orient it locally. Our convention of signs is also given in Figure 6. Note that the type of a curl does not depend on the orientation we choose. The small arcs on the right hand side of these formulas indicate the removal of the curl from the corresponding diagram.

Here is the diagrammatic proof of the behaviour of the binary bracket with a curl in the diagram:

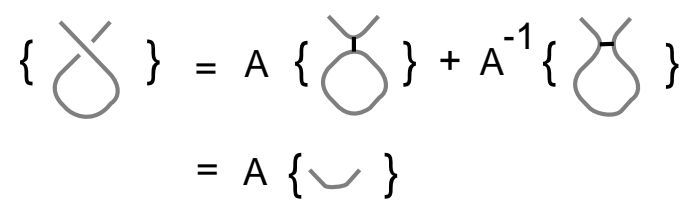

Note that the second diagram contributes zero, since it contains a demand that an arc be colored differently from itself. The proof for the opposite curl goes the same way.

We now make a key observation about the structure of the properly colored states. Note that any link diagram $K$, real or virtual, has an underlying 4-regular plane graph $\operatorname{Sh}(K)$, that we shall call the shadow of $K$. It follows (see Figure 4) from the combinatorics of coloring at a crossing 
that a properly colored state of a diagram $K$ (virtual or classical) is the same as a coloring of the edges of the shadow $\operatorname{Sh}(K)$ with 0 and 1 such that if two edges meet at a vertex and are not adjacent in the cyclic order at that vertex, then they receive different colors. This means that a proper coloring is obtained by walking along the diagram, crossing at each crossing, with a color change at each classical crossing and no change at each virtual crossing. It is easy to see that exactly two such colorings exist for any shadow of a knot diagram. If we have the shadow of a link diagram with an even number of virtual crossings between any two link components, then there are $2^{N}$ proper diagram colorings, where $N$ is the number of link components.

View Figure 5 for an illustration of the coloring statements of the last paragraph for two virtual knots $K$ and $E$. In this figure, we have labeled one of the colored states on shadow diagrams for each knot. The smoothings that correspond to these states are indicated by segments drawn through the crossings. Note that in these virtual cases, there are crossings where the oriented smoothing is different from the smoothing indicated by the colored state. This kind of difference makes it possible for the invariant to detect some virtual knots.

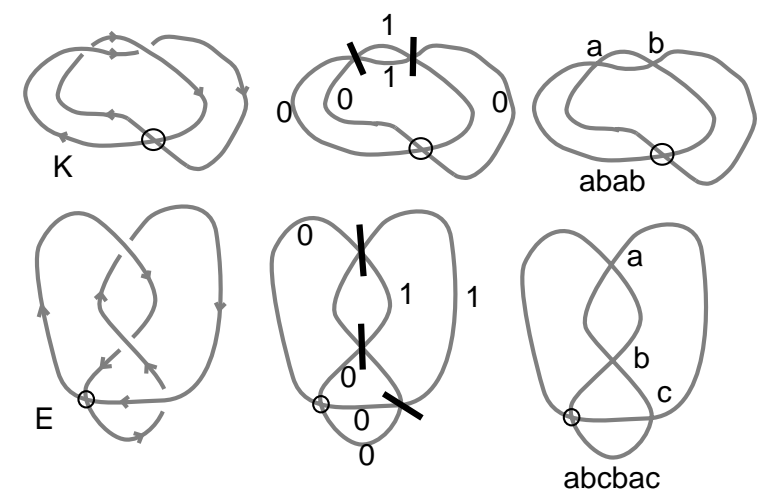

Fig. 5. Virtual trefoil $K$ and virtual figure eight $E$

THEOREM 0. The binary bracket is invariant under regular isotopy for virtual links, and it can be normalized to an invariant of ambient isotopy by the definition

$$
\operatorname{Inv}_{K}(A)=A^{-w(K)}\{K\}(A)
$$

where we choose an orientation for $K$, and where $w(K)$ is the sum of the crossing signs of the oriented link $K$.

$w(K)$ is called the writhe of $K$. The convention for crossing signs is shown in Figure 6. 


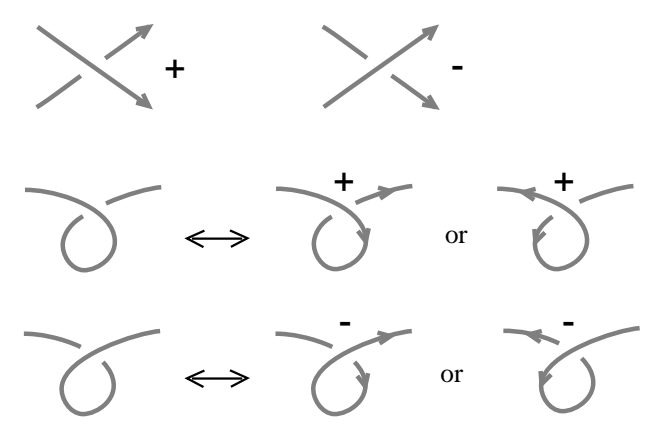

Fig. 6. Crossing signs and curls

Proof of Theorem 0. First we prove invariance under the second Reidemeister move. The diagrammatic proof is:

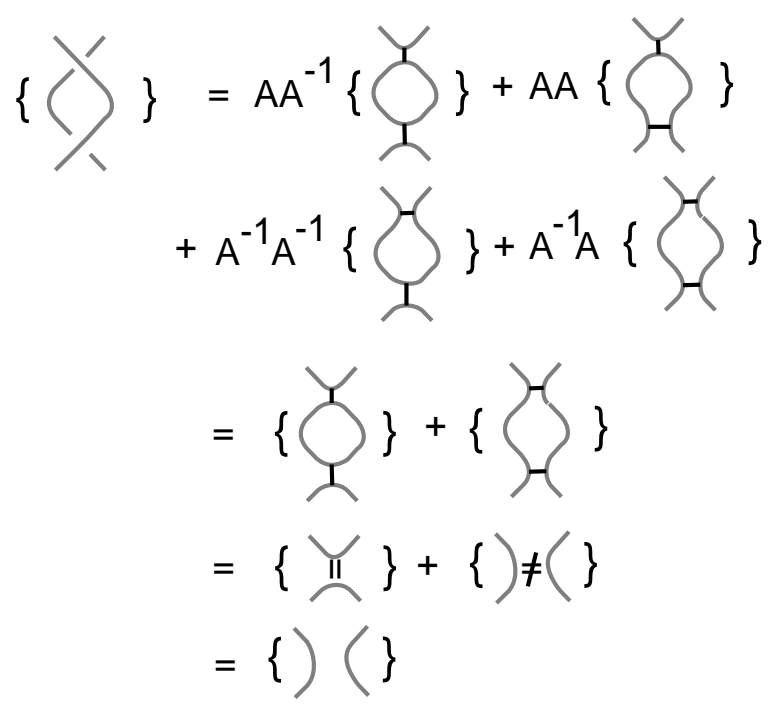

Note that in this expansion the initial second and third terms are zero due to demands for colors to be distinct from themselves. In the remaining two terms, the first consists of two arcs connected through an intermediate circle. If the top arc is colored $X$, then the circle is colored $\sim X(\sim 0=1, \sim 1=0)$ and the bottom arc is hence colored $\sim \sim X=X$. Thus this condition for the first diagram is that the top and bottom arcs have the same color. This is the same as saying that the arcs of the reversed smoothing have the same color. Combined with the statement that the vertical arcs in the second diagram are of different colors, the two diagrams taken together encompass all cases for two vertical arcs. Hence the invariance under the second Reidemeister move is proved.

For invariance under the third Reidemeister move, view Figure 7. 

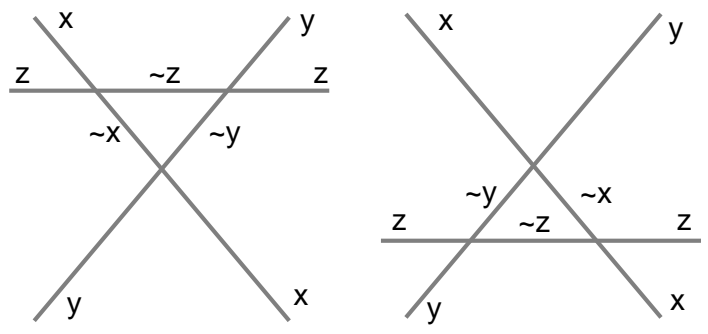

Fig. 7. States for third Reidemeister move

In Figure 7 we illustrate the general pattern of state labels on the shadows of the two sides of the third Reidemeister move. The variables $x, y, z$ take the values 0 or 1 . Note that given choices of the values of these variables for the top free ends of any one of the diagrams, the values on the rest of the diagram are determined by the coloring rule (switch as the signal goes through a classical crossing). Thus we need compare only one state at a time before and after the Reidemeister move. Note further that at a crossing the four labels will be two of one color and two of the other, determining the smoothing corresponding to the state. If we switch all colors at a given crossing, then the smoothing remains the same. Note that before and after the Reidemeister move, corresponding crossings indeed have all their colors switched. The vertex weights are determined by the smoothing and therefore the product of the vertex weights is the same before and after the smoothing. This proves the invariance under the classical Reidemeister third move.

It remains to prove invariance under the moves involving the virtual crossings. This is quite easy and we leave the details to the reader. Writhe normalization works to give invariance under all moves because the writhe itself is an invariant of regular isotopy and invariant under moves involving virtual crossings. This completes the proof of Theorem 0 .

REMARK. The binary bracket can be viewed as an invariant based on the following solution to the Yang-Baxter equation:

$$
R=\left(\begin{array}{cccc}
0 & 0 & 0 & A \\
0 & A^{-1} & 0 & 0 \\
0 & 0 & A^{-1} & 0 \\
A & 0 & 0 & 0
\end{array}\right) .
$$

This $4 \times 4$ matrix is viewed as acting upon a tensor product of a twodimensional space with itself whose basis indices are 0 and 1 . Note that if $A$ is a unit complex number, then $R$ is a unitary matrix. This makes this matrix of interest to us in the context of quantum computing as well as topology. See $[2,10]$. 
The invariant Inv behaves very simply on classical knot and link diagrams.

TheOREM 1. 1. Let $K$ be a classical knot diagram. Then $\{K\}=2 A^{w(K)}$ where $w(K)$ is the writhe of the diagram $K$ (note that for a knot diagram, the writhe is independent of the choice of orientation of that diagram). Thus $\operatorname{Inv}(K)=2$.

2. Let $L$ be a classical link diagram. Then $\{L\}=\sum_{O \in O(L)} A^{w\left(L^{O}\right)}$, where $O(L)$ denotes the set of orientations of $L$, and $L^{O}$ denotes $L$ with the orientation $O$. Thus, for a given orientation $O_{0}$ of $L$ we have

$$
\operatorname{Inv}\left(L^{O_{0}}\right)=\sum_{O \in O(L)} A^{w\left(L^{O}\right)-w\left(L^{O_{0}}\right)} .
$$

Note that if $L$ has components $\left\{L_{1}, \ldots, L_{N}\right\}$, then

$$
w\left(L^{O}\right)-w\left(L^{O_{0}}\right)=\sum_{i<j}\left(\operatorname{Lk}\left(L_{i}^{O}, L_{j}^{O}\right)-\operatorname{Lk}\left(L_{i}^{O_{0}}, L_{j}^{O_{0}}\right)\right),
$$

where $\mathrm{Lk}$ denotes the linking number.

For virtual diagrams the story is quite different, and Inv can be unequal to 2 for virtual knots and unequal to the above writhe or linking number formulas for virtual links.

THEOREM 2. 1. If a link has an odd number of virtual crossings between two of its components, then there is no proper coloring of that link diagram. (See Figure 8 for an illustration of this in the simplest case of a virtual link $H$ with one classical crossing and one virtual crossing. The link $H$ has linking number equal to $1 / 2$, and linking number alone detects its linkedness. By convention the value of an empty sum is zero, and hence $\{H\}=0$, whence $\operatorname{Inv}(H)=0$. Since $\operatorname{Inv}(O O)=4$, we see that Inv detects the linkedness of $H$. This case of empty sums is the first example of the use of Inv to detect virtual links.)

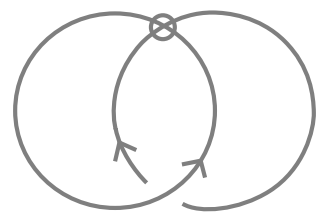

Fig. 8. The link $H$

2. Call a crossing in a virtual knot diagram $K$ odd if, in the Gauss code for that diagram, there are an odd number of appearances of (classical) crossings between the first and the second appearance of $i$. Let

$$
J(K)=w(K) \mid \operatorname{Odd}(K),
$$


where $\operatorname{Odd}(K)$ denotes the collection of odd crossings of $K$, and the restriction of the writhe to $\operatorname{Odd}(K),\left.w(K)\right|_{\operatorname{Odd}(K)}$, means the summation over the signs of the odd crossings in $K$. Then

$$
\operatorname{Inv}(K)=2 A^{-2 J(K)} .
$$

3. If $K$ is a virtual knot, let $K^{*}$ denote the mirror image for $K$ that is obtained by switching all the crossings of the diagram $K$. Then

$$
J\left(K^{*}\right)=-J(K) .
$$

Hence, if $J(K)$ is non-zero, then $K$ is inequivalent to its mirror image.

4. If $K$ is a virtual knot and $J(K)$ is non-zero, then $K$ is not equivalent to a classical knot.

5. Let $L$ be a virtual link diagram. Let $O(L)$ denote the set of orientations of $L$, and $L^{O}$ denote $L$ with orientation $O$. For a given orientation $O_{0}$ of $L$, let

$$
\Sigma\left(L^{O_{0}}\right)=\sum_{O \in O(L)} A^{w\left(L^{O}\right)-w\left(L^{O_{0}}\right)} .
$$

For classical links, $\Sigma\left(L^{O_{0}}\right)=\operatorname{Inv}\left(L^{O_{0}}\right)$. This equality is not always the case for virtual links. Nevertheless, $\Sigma\left(L^{O_{0}}\right)$ is an invariant of virtual links. When these two invariants differ, we can conclude that the virtual link is non-trivial and non-classical. The ratio

$$
\Lambda(L)=\{L\} / \sum_{O \in O(L)} A^{w\left(L^{O}\right)}=\operatorname{Inv}\left(L^{O_{0}}\right) / \Sigma\left(L^{O_{0}}\right)
$$

is an invariant of the unoriented link $L$ that, when not equal to 1 , determines that the link is non-trivial and non-classical.

Remark. View Figure 5. The two virtual knots in this figure illustrate the application of Theorem 2. In the case of the virtual trefoil $K$, the Gauss code of the shadow of $K$ is $a b a b$; hence both crossings are odd, and we have $J(K)=2$. This proves that $K$ is non-trivial, non-classical and inequivalent to its mirror image. Similarly, the virtual knot $E$ has shadow code $a b c b a c$ so that the crossings $a$ and $b$ are odd. Hence $J(E)=2$ and $E$ is also non-trivial, non-classical and chiral. Note that for $E$, the invariant is independent of the type of the even crossing $c$.

Remark. V. Turaev points out to us [13] that implicit in the constructions of his paper on virtual strings [14] are interesting generalizations of the invariant $J(K)$. We shall pursue this topic in another paper.

Proof of Theorem 1. 1. To prove the first part, we note that in a classical knot diagram, there is exactly one state and this state has two proper colorings. The state can be obtained by choosing one coloring of the diagram and smoothing the crossings accordingly. Changing all zeros to ones and all ones 
to zeros gives the other colored state, but does not change the smoothing configuration. We claim that this smoothing configuration can also be obtained by orienting the diagram and forming an oriented smoothing at each crossing. (The resulting state is sometimes referred to as the collection of Seifert circles for the diagram.) To see an example, view Figure 4. The claim follows from the fact that there are an even number of crossings between the first and second occurrence of any given crossing $i$ in the Gauss code of $K$. It follows from this that if (say) the color 0 is the input color to the crossing $i$, then the color 0 will also be the output color of the second appearance of the crossing $i$. The result is that the oriented smoothing of the crossings corresponds to the smoothing designated by the coloring. Given this claim, we need only point out that the state obtained from the oriented smoothings contributes $A^{w(K)}$ to the state summation. This follows directly from the definition of the signs of crossings.

2 . The proof of this second part requires a generalization of the argument we used in the first part. We need to prove the following lemma.

Lemma. Let $\mathcal{C}$ be a collection of Jordan curves in the plane with a set of marked sites (with the structure of a smoothed crossing). We say that $\mathcal{C}$ is properly colored if each curve can be assigned the color 0 or the color 1 such that each site is incident to two distinct colors. Such a proper coloring is possible for $\mathcal{C}$ if and only if it is possible to orient each Jordan curve in $\mathcal{C}$ such that the orientations at each site are parallel to one another.

Proof of Lemma. Consider a collection $\mathcal{C}$ of oriented Jordan curves in the plane. Each curve has a well-defined rotation number that is either plus or minus one. By convention, a clockwise oriented circle has rotation number plus one, while a counterclockwise oriented circle has rotation number minus one. If $C$ is an oriented Jordan curve, let $\operatorname{rot}(C)$ denote its rotation number. Each curve in $\mathcal{C}$ also has a depth $d(C)$ defined to be $d(C)=$ the least number of transverse crossings with curves in $\mathcal{C}$ that are needed to draw an arc from the interior of $C$ to the unbounded region in the plane. For example, if a curve $C_{1}$ surrounds another curve $C_{2}$ with some pair of arcs from the two curves adjacent to one another, then $d\left(C_{2}\right)=1+d\left(C_{1}\right)$. In a nest of $n$ circles, the innermost circle has depth $n$. A curve drawn in the unbounded region has depth 0 .

Now define for each curve $C$ in $\mathcal{C}$ the function

$$
\lambda(C)=(-1)^{d(C)} \operatorname{rot}(C) .
$$

It is then easy to see that two adjacent curves $C_{1}$ and $C_{2}$ in $\mathcal{C}$ have parallel orientations if and only if $\lambda\left(C_{1}\right) \neq \lambda\left(C_{2}\right)$. In Figure 9 we illustrate three curves with locally parallel orientations. Note that the two concentric curves have the same rotation number, while the two adjacent but not concentric 


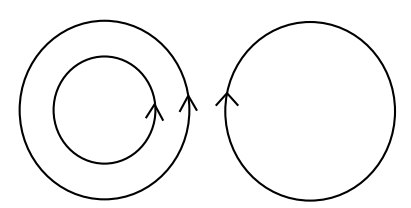

Fig. 9. Nested and adjacent oriented circles

curves have opposite rotation number. The Lemma follows from this observation. (We label a curve $C$ with $(1+\lambda(C)) / 2$ to change to labels of 0 and 1 from labels of -1 and +1 .)

With this lemma in hand, we see that every properly colored state of a classical link diagram corresponds to an orientation of that diagram, and that the evaluation of that state contributes $A$ raised to the writhe of that choice of orientation. Once the coloring along any given link component is chosen, there is a unique choice of labeling for the rest of the link diagram to produce a given orientation. The formulas for part 2 of the theorem follow directly from these observations.

This completes the proof of Theorem 1.

Proof of Theorem 2. Let $K$ be a virtual knot. Just as in the classical case, there are only two labeled states for $K$. Each state is obtained by consecutively labeling the diagram with zeros and ones so that arcs separated by classical crossings are oppositely labeled. Consider the Gauss code for $\operatorname{Sh}(K)$. Without loss of generality, we can assume that the orientation of $K$ is coincident with the order of the Gauss code. Let $i$ denote one of the classical crossings in $\operatorname{Sh}(K)$. We claim that the oriented smoothing at $i$ is identical with the state smoothing at $i$ if and only if the crossing $i$ is even (see the definition of even and odd crossings given above). To see this claim, view Figure 10. In this figure we illustrate the case of an even crossing where there are zero classical crossings between the first and second appearance of $i$. The local configuration of colors is only changed by changing the parity of the number of classical crossings between the first and second appearance of $i$, and we see that in the even case the state smoothing is coincident with the oriented smoothing.

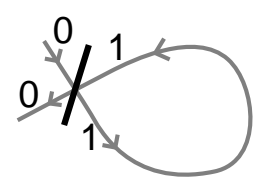

Fig. 10. An even crossing

We know, therefore, that $K=2 A^{a-b}$, where $a$ is the sum of the signs of the even crossings and $b$ is the sum of the signs of the odd crossings. Note 
that $w(K)=a+b$. By definition $J(K)=b$. Hence

$$
\operatorname{Inv}(K)=2 A^{a-b-w(K)}=2 A^{a-b-a-b}=2 A^{-2 b}=2 A^{-2 J(K)} .
$$

This completes the proof of the formula stated in the theorem. It is clear that changing all the crossings in the knot reverses the sign of $J(K)$. Since $\operatorname{Inv}(K)=2$ for classical knots, we see that $J(K)$ detects non-classicality whenever it is non-zero. The fifth statement in this theorem is immediately obvious from the preceding discussion. This completes the proof of Theorem 2.

4. Examples. In this section we give a sampling of examples that illustrate the use of the binary bracket polynomial and the associated self-linking invariant for virtual knots.

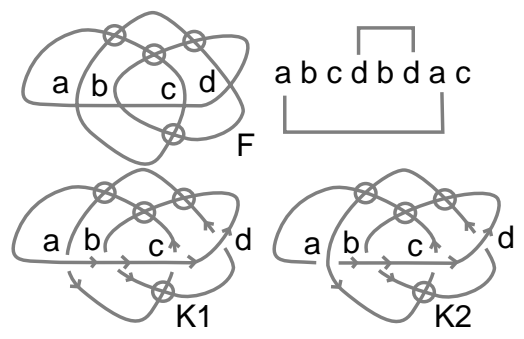

Fig. 11. Two knots

In Figure 11 we show virtual knots $K_{1}$ and $K_{2}$. Both knots have underlying flat Gauss code $a b c d b d a c$. The code is odd for vertices $a$ and $d$. Thus $J\left(K_{1}\right)=0$ and $J\left(K_{2}\right)=2$. The invariant $J(K)$ tells us nothing about $K_{1}$, but it does tell us that $K_{2}$ is non-classical and not equivalent to its mirror image. An independent calculation, that we omit, shows that $K_{1}$ has unit Jones polynomial, but that it is detected by the two-stand Jones polynomial.

In Figure 12 we illustrate a persistent virtual tangle $T$. It follows from the $J$-invariant that whenever this tangle occurs in a virtual knot diagram $K$ with no other virtual crossings except those in the tangle $T$, then this diagram is non-trivial, non-classical and inequivalent to its planar mirror image. The proof of this statement is inherent in the figure. To see this note that we have indicated a schematic version of the general code for some diagram in which the tangle sits. The code has the form

$$
A 1 * 2 * 34 B * 341 * 2,
$$

where the $*$ denotes the occurrence of a virtual crossing in the diagram for the tangle $T$, and $A$ and $B$ are strings for the remaining part of the Gauss code of $K$. Since a crossing is odd exactly when its pair of appearances in 

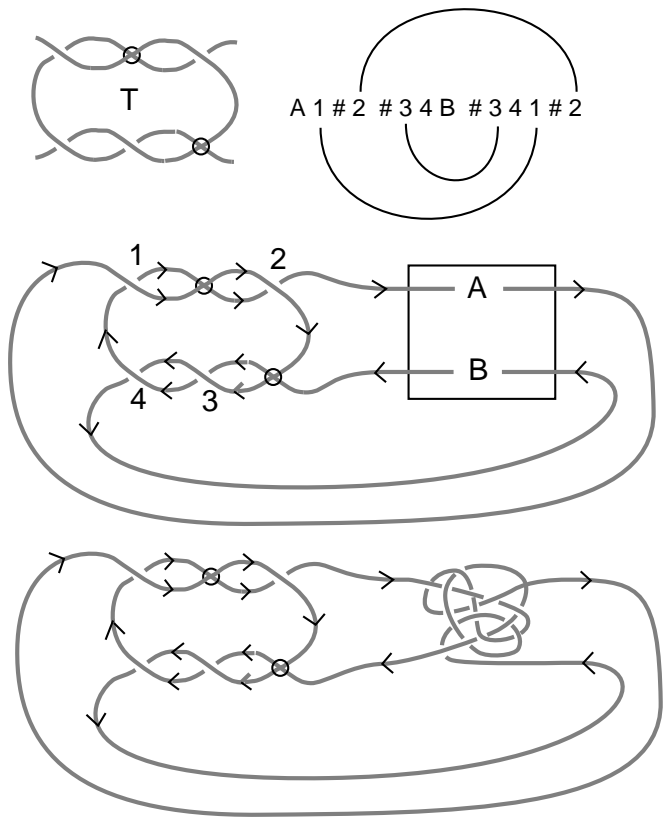

Fig. 12. A persistent virtual tangle

the Gauss code contains an odd number of virtual crossings, it follows that the only odd crossings in the diagram $K$ are 1,2 and 3. Hence $J(K)=3$, proving the result.
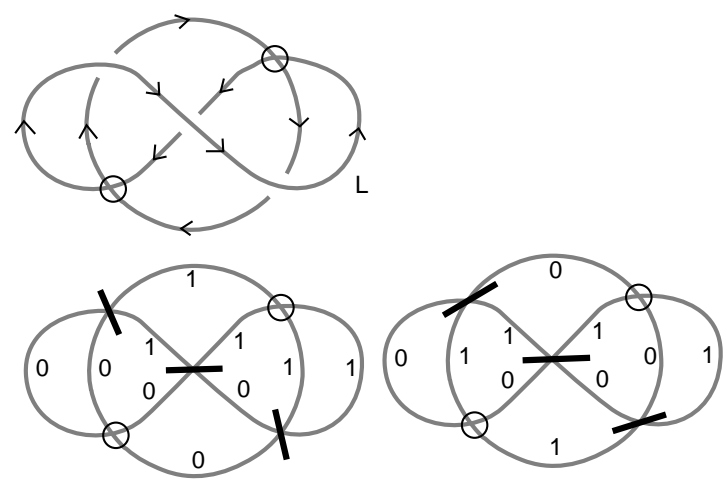

Fig. 13. Virtual Whitehead link

In Figure 13 we show a "virtual Whitehead link" L. (The Whitehead link is a classical non-trivial link of two components with linking number zero.) The link $L$ has $w(L)=-1$ and this is true for each of its four orientations. Hence $\sum_{O \in O(L)} A^{w\left(L^{O}\right)}=4 A^{-1}$. The two state diagrams in Figure 13 show 
that $\{L\}=2\left(A^{-1}+A^{3}\right)$. Thus

$$
\Lambda(L)=\{L\} / \sum_{O \in O(L)} A^{w\left(L^{O}\right)}=\left(A^{-1}+A^{3}\right) / 2 A^{-1}=\left(1+A^{4}\right) / 2 .
$$

Since $\Lambda(L)$ is not equal to 1 , we conclude that the unoriented link $L$ is not trivial and not classical. Since $\Lambda(L)(A) \neq \Lambda(L)\left(A^{-1}\right)$, we conclude that $L$ is not equivalent to its planar mirror image.

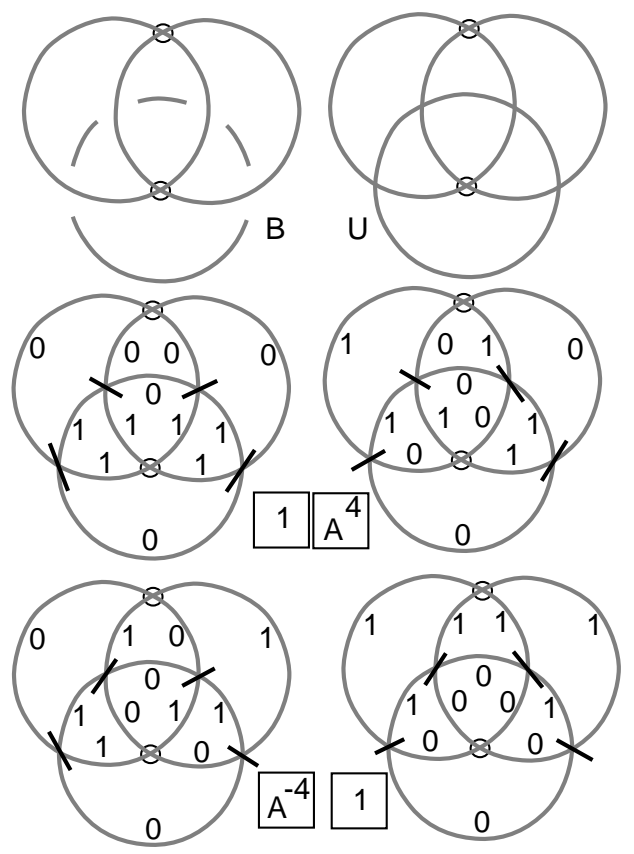

Fig. 14. Virtual Borrommean rings

In Figure 14 we show a link $B$ that could be called the "virtual Borrommean rings". Note that $B$ has writhe zero for each of its orientations. Thus $\sum_{O \in O(B)} A^{w\left(B^{O}\right)}=4$. The states illustrated in the figure show that $\{L\}=2\left(2+A^{4}+A^{-4}\right)$. Thus

$$
\Lambda(B)=\{B\} / \sum_{O \in O(B)} A^{w\left(B^{O}\right)}=\left(2+A^{4}+A^{-4}\right) / 2 .
$$

This shows that the virtual Borrommean rings are non-trivial and nonclassical.

5. Colorings and generalizations. It is natural to ask what happens in the formalism of the binary bracket if we replace coloring by two colors with colorings by an arbitrary number of colors. That is, we ask about $\{K\}_{n}$, where this $n$-ary bracket evaluation satisfies the equations below. 


$$
\begin{gathered}
\{\searrow\}_{\mathrm{n}}=\mathrm{A}\{\bigwedge\}_{\mathrm{n}}+\mathrm{B}\{\supset-C\}_{\mathrm{n}}, \\
\{K \amalg O\}_{n}=n\{K\}, \quad\{O\}_{n}=n .
\end{gathered}
$$

Here our conventions are the same as before and $\{K\}_{n}$ gives a well defined polynomial on virtual link diagrams, in the commuting variables $A$ and $B$. It appears, however, that unless $n=2$ there is no way to obtain non-trivial invariants of (virtual) knots and links from this scheme. Nevertheless, it is of interest to consider the underlying problem of coloring virtual link diagrams according to the generalization of our rules that is inherent in these equations.

For this purpose, we define a specialized $n$-ary shadow bracket, by the following equations.

$$
\begin{gathered}
{[X]_{\mathrm{n}}=[Y]_{\mathrm{n}}+[\supset-C]_{\mathrm{n}},} \\
{[K \amalg O]_{n}=n[K], \quad[O]_{n}=n .}
\end{gathered}
$$

We call this evaluation of a virtual shadow diagram (note that the crossing is neither over nor under) the shadow bracket to emphasize that the crossings in the diagram are flattened. Note that we still have virtual crossings and flat classical crossings. Coloration at a flat classical crossing follows the rules indicated by the shadow bracket. That is, the rule for coloring is that as one crosses a crossing the color must change, and there are exactly two distinct colors at any given flat crossing. See Figure 15. At a virtual crossing colors

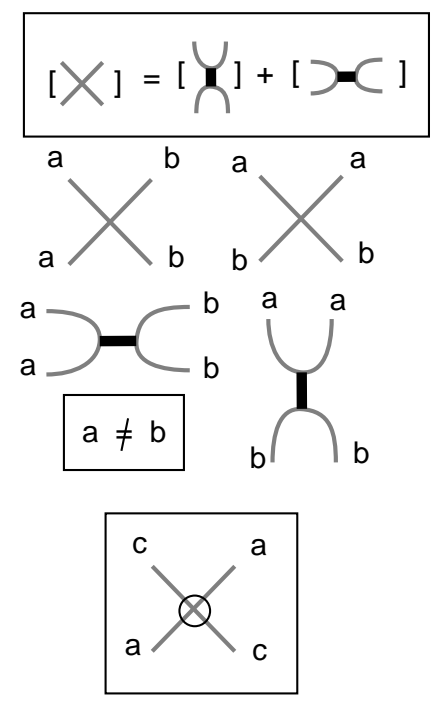

Fig. 15. Coloring rules at flat and virtual crossings 
do not change when one crosses the crossing and either one or two colors are present at the virtual crossing. The equation above for the shadow bracket can be read as tautological. Any coloring at a given crossing must be in one of the two disjoint possibilities indicated. The value of the shadow bracket on a flat virtual diagram is equal to the number of colorings of the diagram that are possible under these rules for $n$ colors.

We would like to know which virtual diagrams are colorable in $n$ colors for $n$ greater than two. When $n$ is equal to two, the answer is simple, and already used in the virtual knot theory part of this paper. A virtual diagram is colorable with two colors whenever the number of virtual crossings shared between any two components of the diagram is even. The situation for higher $n$ is much more subtle. First of all consider the diagram in Figure 16. This diagram is the projection of the virtual Hopf link of Figure 8. It is uncolorable for any $n$, since its structure demands colors that are unequal to themselves.

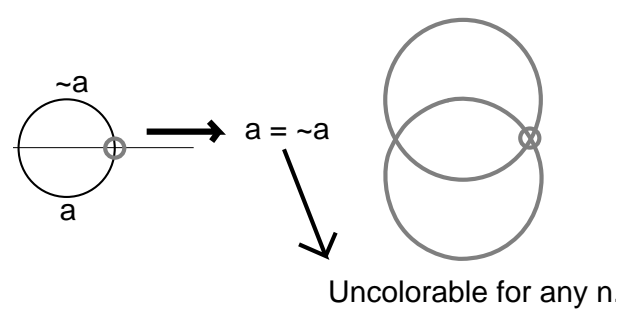

Fig. 16. The simplest uncolorable

On the other hand, consider the diagram in Figure 17. The reader will have no difficulty in verifying that this diagram can be colored in three colors but not in two colors.

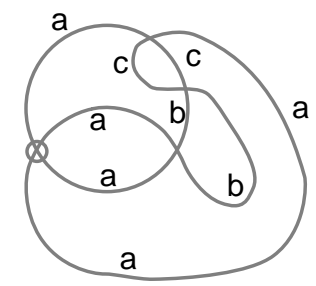

Fig. 17. A diagram that needs three colors

In the top line of Figure 18 we give an example of a more complex diagram that is uncolorable for any $n$. Note that an uncolorable diagram will of necessity have the structure of a flat link diagram that has an odd number of virtual crossings between some of its components. But it is a subtle matter to characterize the uncolorability. 


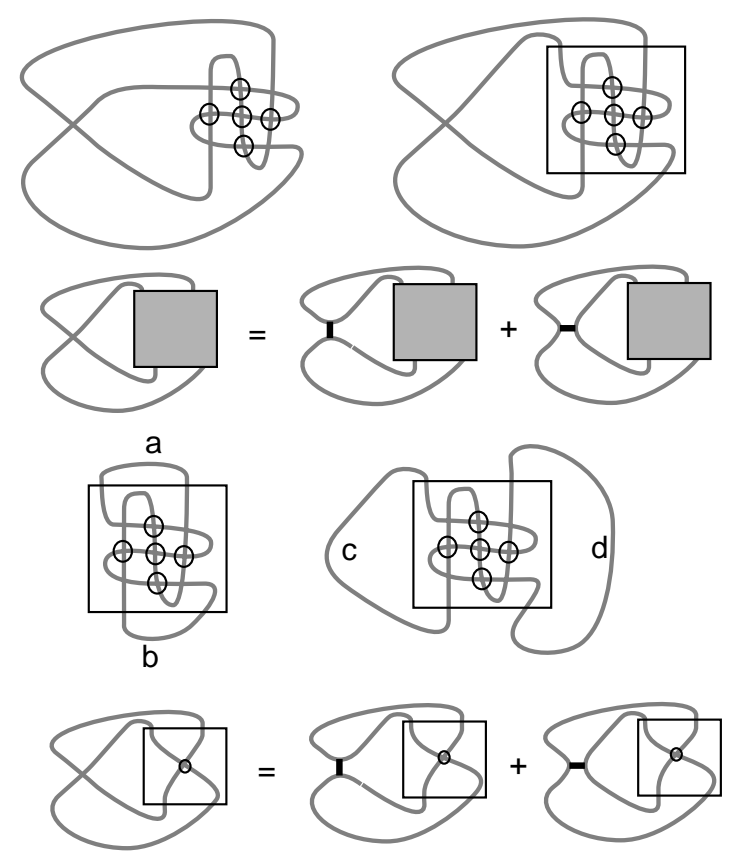

Fig. 18. Form of an uncolorable diagram

One way to begin to understand uncolorables is to look at the expansion of the shadow bracket for one crossing. View Figure 18. In this figure we illustrate the basic expansion equation for a diagram at one crossing, with the rest of the diagram concentrated in a tangle box with four external arcs. Uncolorability of $G$ implies that two ways of connecting the arcs on the tangle box give graphs that, if colorable, force the same color on the two external arcs resulting from the connection. In the case of the examples shown in Figure 18, it is not hard to see that they satisfy this condition.

The problem of classifying exactly which shadow diagrams are colorable appears to be quite interesting. In fact, it is related directly to the classical four color problem $[9,6]$. We now explain this connection.

5.1. Cubic graphs, shadow diagrams and the four color problem. A graph is said to be cubic if there are locally three edges per node. Graphs are allowed to have loops and to have multiple edges between two nodes. A cubic map $G$ is said to be properly colored with $n$ colors if the edges of $G$ are colored from the $n$ colors so that all colors incident to any node of $G$ are distinct. It is well known that the following theorem is equivalent to the famous Four Color Theorem for maps in the plane.

Theorem (equivalent to the Four Color Theorem). Let $G$ be a connected cubic plane graph with no isthmus (an isthmus is an edge whose 
deletion disconnects the graph). Then $G$ is properly 3-colorable (as defined above).

We shall first use this result to give yet another (well known) equivalent version of the Four Color Theorem (FCT). To this end, call a disjoint collection $\mathcal{E}$ of edges of $G$ that includes all the vertices of $G$ a perfect matching of $G$. Then $\mathcal{C}(\mathcal{E}, \mathcal{G})=G$-Interior $(\mathcal{E})$ is a collection of cycles (graphs homeomorphic to the circle, with two edges incident to each node). We say that $\mathcal{E}$ is an even perfect matching of $G$ if every cycle in $\mathcal{C}(\mathcal{E})$ has an even number of edges.

TheOREM. The following statement is equivalent to the Four Color Theorem: Let $G$ be a plane cubic graph with no isthmus. There there exists an even perfect matching of $G$.

Proof. Let $G$ be a cubic plane graph with no isthmus. Suppose that $G$ is properly 3 -colored from the set $\{a, b, c\}$. Let $\mathcal{E}$ denote all edges in $G$ that receive the color $c$. Then, by the definition of proper coloring, the edges in $\mathcal{E}$ are disjoint. By the definition of proper 3-coloring every node of $G$ is in some edge of $\mathcal{E}$. Thus $\mathcal{E}$ is a perfect matching of $G$. Since each cycle in $\mathcal{C}(\mathcal{E}, \mathcal{G})$ is two-colored by the set $\{a, b\}$, each cycle is even. Hence $\mathcal{E}$ is an even perfect matching of $G$.

Conversely, suppose that $\mathcal{E}$ is an even perfect matching of $G$. Then we may assign the color $c$ to all the edges of $\mathcal{E}$, and color the cycles in $\mathcal{C}(\mathcal{E})$ using $a$ and $b$ (since each cycle is even). The result is a proper 3 -coloring of the graph $G$. This completes the proof of the theorem.
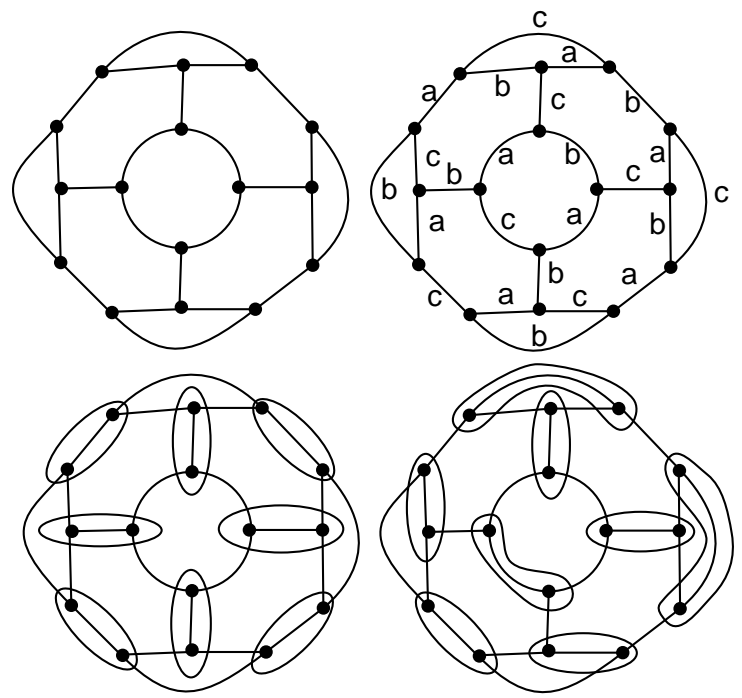

Fig. 19. Perfect matchings of a cubic plane graph 
Remark. See Figure 19 for an illustration of two perfect matchings of a graph $G$. One perfect matching is not even. The other perfect matching is even, and the corresponding coloring is shown. This theorem shows that one could conceivably divide the proving of the FCT into two steps: First prove that every cubic plane isthmus-free graph has a perfect matching. Then prove that it has an even perfect matching. In fact, the existence of a perfect matching is hard, but available [4], while the existence of an even perfect matching is really hard!

Proposition. Every cubic graph with no isthmus has a perfect matching.

Proof. See [4, Chapter 4],

REMARK. There are graphs that are uncolorable. Two famous such culprits are indicated in Figure 20. These are examples of graphs with perfect matchings, but no even perfect matching. The second example in Figure 20 is the "dumbell graph". It is planar, but has an isthmus. The first example is the Petersen graph. This graph is non-planar. We have illustrated the Petersen with one perfect matching that has two five cycles. No perfect matching of the Petersen is even. The third "double dumbell" graph illustrated in Figure 20 has no perfect matching.

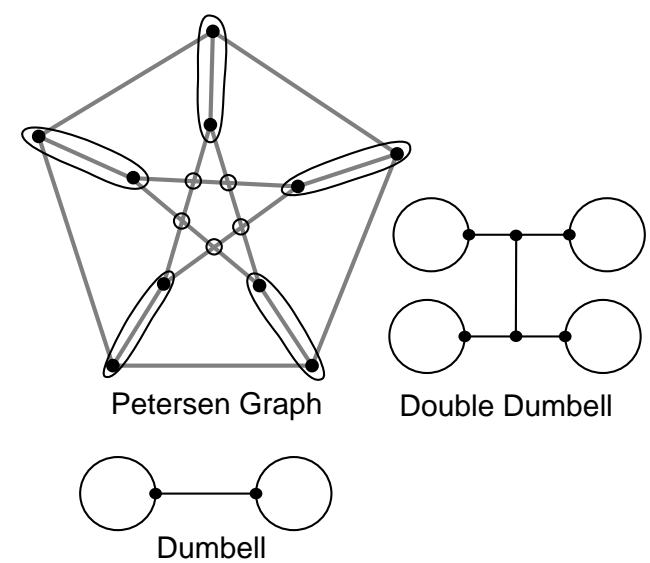

Fig. 20. Dumbbell and Petersen

We are now in a position to explain the relationship between coloring cubic graphs and coloring virtual shadow diagrams. Let $G$ be a cubic plane graph without isthmus. We shall say that a graph with no isthmus is bridgeless. Let $\mathcal{E}$ be a perfect matching for $G$. Replace each edge in $\mathcal{E}$ by the combination of flat crossing and virtual crossing shown in Figure 21. Call the resulting flat virtual diagram $D(G, \mathcal{E})$. We have the following theorem. 
TheOREM. Let $G$ be a bridgeless cubic plane graph. Let $\mathcal{E}$ be a perfect matching for $G$. Then $G$ can be properly colored with 3 colors if and only if $D(G, \mathcal{E})$ can be properly colored with 3 colors as a flat virtual diagram. The Four Color Theorem is equivalent to the statement: There exists a perfect matching for $G$ such that $D(G, \mathcal{E})$ can be colored with two colors. That is, the binary bracket evaluated at $A=1$ does not vanish for $D(G, \mathcal{E})$.

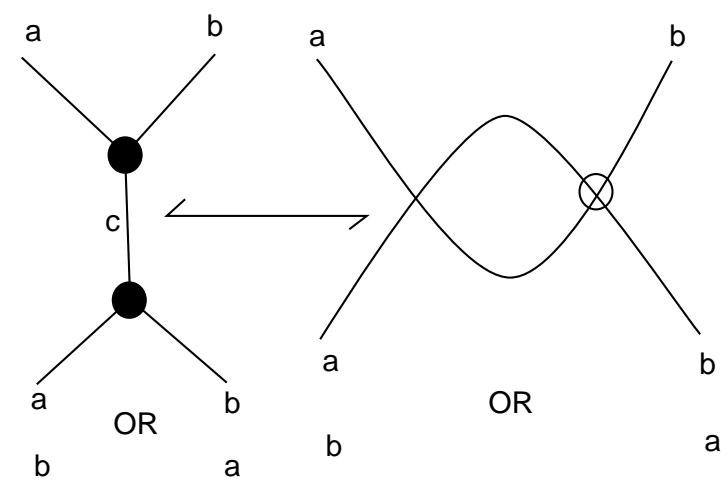

Fig. 21. Translation between cubic graphs and shadow virtual diagrams

Proof. As is shown in Figure 21, the coloring conditions for the double $\mathrm{Y}$ configuration and for the replacement shadow diagram are the same when one is coloring at $n=3$. Note that the edge that is deleted in passing to the shadow diagram will receive the third color that is distinct from the two colors that appear at the flat crossing in the shadow diagram. (This shows why this correspondence will not work for $n$ greater than 3.) Using the perfect matching, one can replace each edge in $\mathcal{E}$ with the corresponding shadow diagram configuration. The result is a virtual shadow diagram whose colorings are in one-to-one correspondence with the colorings of the original graph. The rest of the theorem follows from our discussion of perfect matchings and the need for an even perfect matching to satisfy the coloring condition at all vertices of the graph. With an even perfect matching, the corresponding shadow diagram can be colored with two colors. Hence its binary bracket at $A=1$ does not vanish. This completes the proof.

REMARK. There is much more to explore in this domain. In Figure 22 we illustrate how the translation process from cubic graphs to virtual shadow diagrams takes a version of the Petersen graph, with a specific perfect matching to the uncolorable shadow diagram at the top of Figure 18 (after removal of two redundant virtual crossings). In general, any virtual shadow diagram can be translated into a cubic graph (with some perfect matching) by placing two canceling virtual crossings next to any isolated flat crossing in the diagram and then using the combination of flat crossing and virtual crossing 


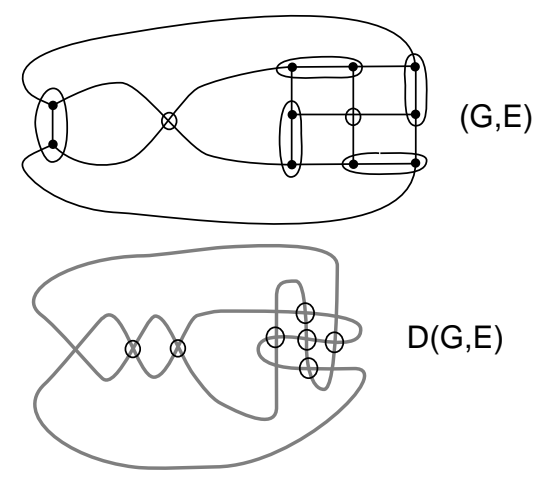

Fig. 22. Petersen graph $G$ with perfect matching $E$, and virtual shadow diagram $D(G, E)$

to form a double $\mathrm{Y}$ configuration. The resulting cubic graph may or may not be planar as a result of this operation. For any cubic graph $G$ with no isthmus, each perfect matching of $G$ gives rise to a virtual shadow diagram. Thus there is a multiplicity of virtual shadow diagrams corresponding to a given cubic graph. Note that for $n$ greater than 3 the colorings for cubic graphs and the colorings for virtual shadow diagrams are no longer in one-to-one correspondence (since in that case the top and bottom ends of the double Y can receive different pairs of colors). We shall reserve further comments on this colorful domain for the next paper.

\section{References}

[1] J. S. Carter, S. Kamada and M. Saito, Stable equivalence of knots on surfaces and virtual knot cobordisms, J. Knot Theory Ramifications 11 (2002), 311-320.

[2] H. A. Dye, Unitary solutions to the Yang-Baxter equation in dimension four, Quantum Inf. Processing 2 (2002), 117-151; arXiv:quant-ph/0211050

[3] M. Goussarov, M. Polyak and O. Viro, Finite type invariants of classical and virtual knots, Topology 39 (2000), 1045-1068.

[4] D. A. Holton and J. Sheehan, The Petersen Graph, Austral. Math. Soc. Lecture Ser. 7, Cambridge Univ. Press, Cambridge, 1993.

[5] L. H. Kauffman, State models and the Jones polynomial, Topology 26 (1987), 395407.

[6] -, Knots and Physics, 3rd ed., World Sci., Singapore, 2001.

[7] —, Virtual knot theory, European J. Combin. 20 (1999), 663-690.

[8] - Detecting virtual knots, Atti Sem. Mat. Fis. Univ. Modena 49 (2001), suppl., 241-282.

[9] - Reformulating the map color theorem, in: Accota Graph Theory proceedings, to appear; arXiv:math.CO/0112266.

[10] L. H. Kauffman and S. J. Lomonaco Jr., Braiding operators are universal quantum gates, arXiv:quant-ph/0401090.

[11] G. Kuperberg, What is a virtual link?, Algebr. Geom. Topol. 3 (2003), 587-591; arXiv:math.GT/0208039. 
[12] S. Nelson, Unknotting virtual knots with Gauss diagram forbidden moves, J. Knot Theory Ramifications 10 (2001), 931-935.

[13] V. Turaev, private conversation.

[14] —, Virtual strings and their cobordisms, arXiv:math.GT/0311185.

Department of Mathematics, Statistics and Computer Science University of Illinois at Chicago

851 South Morgan St.

Chicago, IL 60607-7045, U.S.A.

E-mail: kauffman@uic.edu

Received 4 May 2004;

in revised form 29 October 2004 